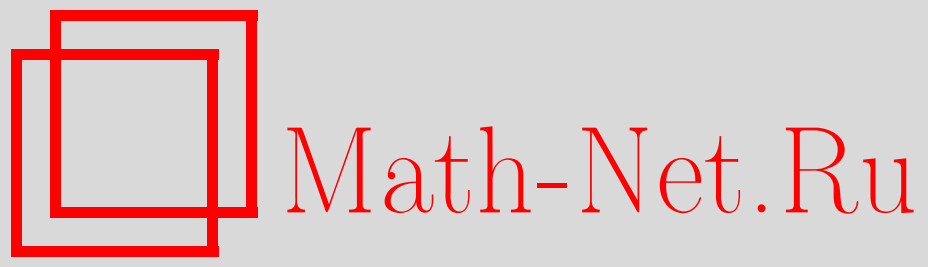

А. В. Селиванов, О мартингальных мерах в экспоненциальных моделях Леви, Теория вероятн. и ее примен., 2004, том 49, выпуск 2, 317-334

DOI: https://doi.org/10.4213/tvp221

Использование Общероссийского математического портала Math-Net.Ru подразумевает, что вы прочитали и согласны с пользовательским соглашением

http://www . mathnet.ru/rus/agreement

Параметры загрузки:

IP: 18.234 .197 .8

26 апреля 2023 г., 09:26:48

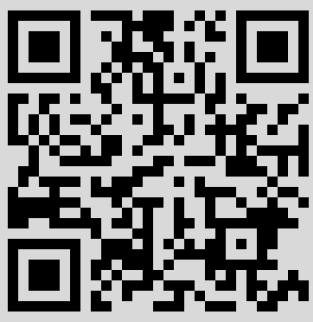


(c) 2004 г.

\author{
СЕЛИВАНОВ А. В.*
}

\title{
О МАРТИНГАЛЬНЫХ МЕРАХ В ЭКСПОНЕНЦИАЛЬНЫХ МОДЕЛЯХ ЛЕВИ
}

В работе изучается проблема существования и единственности мартингальных мер в экспоненциальных моделях Леви вида

$$
S_{t}=e^{X_{t}}, \quad S_{t}=e^{X_{\tau_{t}}},
$$

где $X$ - процесс Леви, $\tau$ - неубываюший процесс, не зависящий от $X$.

Ключевые слова и фразы: фундаментальная теорема теории арбитража, экспоненциальная модель Леви, мартингальная мера, сигма-мартингальная мера, равномерно интегрируемая мартингальная мера.

\section{1. Введение}

В финансовой математике важную роль играет вопрос существования и единственности мартингальной меры в различных моделях цен. Это вопрос о наличии арбитража и полноте в соответствующей модели рынка (см. [11, гл. V, VII]).

В данной работе мы даем ответ на этот вопрос для следующих двух моделей цен, популярных в современной финансовой математике (см. [2], [3], [9], [11]).

1. Экспоненциальная модель Леви, т.е. модель, в которой процесс дисконтированной цены имеет вид

$$
S_{t}=e^{X_{t}}
$$

где $X$ - процесс Леви.

2. Экспоненциальная модель Леви с заменой времени, т.е. модель, в которой процесс дисконтированной цены имеет вид

$$
S_{t}=e^{(X \circ \tau)_{t}}
$$

* Московский государственный университет им. М.В. Ломоносова, механикоматематический факультет, Ленинские горы, 119992 Москва, Россия; е-mail: a_seliv@mech.math.msu.su 
где $X$ - процесс Леви, $\tau$ - неубывающий процесс, не зависящий от $X$, $(X \circ \tau)_{t}=X_{\tau_{t}}$ (эта модель была введена в [3]).

Мы рассмотрим случаи как конечного, так и бесконечного временного горизонта.

Классы мартингальных мер, которые мы будем изучать, - это классы, возникающие в фундаментальной теореме теории арбитража (ФТТА). Для случая непрерывного времени эта теорема может быть сформулирована разными способами. Предположим, что процесс дисконтированной цены $S$ является семимартингалом.

В традиционной ФТТА, полученной $\Phi$. Делбаеном и В. Шахермайером в [6], утверждается, что в модели $\left(\Omega, \mathscr{F},\left(\mathscr{F}_{t}\right)_{t \in[0, T]}, \mathbf{P},\left(S_{t}\right)_{t \in[0, T]}\right)$ отсутствует бесплатный ленч с исчезающим риском (или, эквивалентно, бесплатный ленч с ограниченньл риском) тогда и только тогда, когда $\mathscr{M}_{\sigma} \neq \varnothing$, где

$$
\mathscr{M}_{\sigma}=\left\{\mathbf{Q} \sim \mathbf{P}: S \text { является }\left(\mathscr{F}_{t}, \mathbf{Q}\right)-\sigma \text {-мартингалом }\right\}
$$

(элемент из $\mathscr{M}_{\sigma}$ называется $\sigma$-мартингальной мерой). Этот же результат справедлив и для модели с бесконечным временным горизонтом.

Однако понятие арбитража может быть введено иначе, чем это делается в [6] (см. [11, гл. VII, § 2]). В некоторых случаях (см., например, [14]) ФТТА для моделей с конечным временным горизонтом формулируется с использованием множества

$$
\mathscr{M}=\left\{\mathbf{Q} \sim \mathbf{P}: S \text { является }\left(\mathscr{F}_{t}, \mathbf{Q}\right) \text {-мартингалом }\right\}
$$

(элемент из $\mathscr{M}$ называется мартингальной мерой).

В силу некоторых соображений оказывается полезным рассматривать следуюшее множество для моделей с бесконечным временным горизонтом:

$$
\begin{aligned}
& \mathscr{U} \mathscr{M}=\{\mathbf{Q} \sim \mathbf{P}: S \text { является }\left(\mathscr{F}_{t}, \mathbf{Q}\right) \text {-равномерно } \\
&\text { интегрируемым мартингалом }\}
\end{aligned}
$$

(элемент из $\mathscr{U} \mathscr{M}$ называется равномерно интегрируемой мартингальной мерой).

Заметим, что множества $\mathscr{M}$ и $\mathscr{U} \mathscr{M}$ могут быть рассмотрены вместо $\mathscr{M}_{\sigma}$ в традиционной ФТТА при некоторых дополнительных ограничениях на модель (см. [5]).

В этой работе мы изучаем проблему сушествования и единственности $\sigma$-мартингальной, мартингальной и равномерно интегрируемой мартингальной меры для моделей (1.1) и (1.2) с конечным и бесконечным временным горизонтом. Единственная нерешенная проблема - это изучение $\mathscr{M}_{\sigma}$ для модели (1.2) с бесконечным временным горизонтом (см. таблицу 1$)$. 


\begin{tabular}{|c|c|c|c|c|}
\hline \multirow[t]{2}{*}{ Модель } & \multicolumn{2}{|c|}{$\begin{array}{c}\text { Конечный } \\
\text { временной горизонт }\end{array}$} & \multicolumn{2}{|c|}{$\begin{array}{c}\text { Бесконечный } \\
\text { временной горизонт }\end{array}$} \\
\hline & $\mathscr{M}_{\sigma}$ & $\mathscr{M}$ & $\mathscr{M}_{\sigma}$ & $\mathscr{U} \mathscr{M}$ \\
\hline$S_{t}=e^{X_{t}}$ & 3.1 & 3.2 & 4.1 & 4.2 \\
\hline$S_{t}=e^{(X \circ \tau)_{t}}$ & 5.1 & 5.2 & & 6 \\
\hline
\end{tabular}

Таблица 1. Структурная таблица моделей, рассматриваемых в работе. Номер в ячейке означает пункт, в котором рассматривается соответствующая модель. Например, класс $\sigma$-мартингальных мер для модели (1.1) с конечным временным горизонтом исследуется в п. 3.1.

\section{2. Вспомогательные определения и результаты}

В этом разделе мы приводим некоторые определения и результаты, которые будут использованы в дальнейших рассуждениях.

2.1. Процессы Леви. Пусть $h$ - функция усечения, т.е. ограниченная функция из $\mathbf{R}$ в $\mathbf{R}$ такая, что $h(x)=x$ в некоторой окрестности нуля. Обозначение $X \sim(b, c, \nu)_{h}$ означает, что $X$ - это процесс Леви с характеристиками $(b, c, \nu)$ относительно функции усечения $h$, т.е.

$$
\mathbf{E} e^{i \lambda X_{t}}=\exp \left\{t \cdot\left[i \lambda b-\frac{\lambda^{2}}{2} c+\int_{\mathbf{R}}\left(e^{i \lambda x}-1-i \lambda h(x)\right) \nu(d x)\right]\right\}, \quad t \geqslant 0
$$

(если $\int_{-1}^{1}|x| \nu(d x)<\infty$, мы можем взять $h=0$ ). Всюду далее $H$ обозначает каноническую функцию усечения, т.е. $H(x)=x I(|x| \leqslant 1)$. Понятие Q-мартингала (локального, $\sigma$-мартингала) означает, что процесс является Q-мартингалом (локальным, $\sigma$-мартингалом) на своей натуральной фильтрации.

Предложение 2.1. Пусть $X$ - прочесс Леви с характеристиками $(b, c, \nu)_{h}$. Предположим, что $\mathbf{E} e^{X_{1}}<\infty$. Тогда

(i) для любого $t \geqslant 0$ мы имеем

$$
\mathbf{E} e^{X_{t}}=\exp \left\{t \cdot\left[b+\frac{c}{2}+\int_{\mathbf{R}}\left(e^{x}-1-h(x)\right) \nu(d x)\right]\right\}
$$

(ii) $e^{X}$ - мартингал (относительно своей натуральной Фильтраиии) тогда и только тогда, когда

$$
b+\frac{c}{2}+\int_{\mathbf{R}}\left(e^{x}-1-h(x)\right) \nu(d x)=0 .
$$

Док а з а тель с т в о. (i) См. [10, теорема 25.17] или [11, гл. VII, § 3c, п. 4].

(ii) Это утверждение следует из (i). 
Предложение 2.2. Пусть $X$ - прочесс Леви с характеристиками $(b, c, \nu)_{h}$. Прочесс $X$ является неубьваюоим тогда и только тогдa, когда $c=0, \nu((-\infty, 0))=0, \int_{(0,1]} x \nu(d x)<\infty u b-\int_{\mathbf{R}_{+}} h(x) \nu(d x) \geqslant 0$.

Доказательство см. в [10, теорема 21.5].

Предложение 2.3. Пусть $\left(X_{t}\right)_{t \in[0, T]}-$ прочесс Леви по мере $\mathbf{P} c$ характеристиками $(b, c, \nu)_{h}$. Пусть число $\lambda$ таково, что

$$
\int_{\mathbf{R}}\left|e^{\lambda x}\left(e^{x}-1\right)-h(x)\right| \nu(d x)<\infty
$$

$u$

$$
b+\left(\lambda+\frac{1}{2}\right) c+\int_{\mathbf{R}}\left(e^{\lambda x}\left(e^{x}-1\right)-h(x)\right) \nu(d x)=0 .
$$

Определим меру $\mathbf{P}^{\lambda}=k e^{\lambda X_{T}} \mathbf{P}$, где $k-$ нормирующая постоянная. Тогда прочесс $\left(e^{X_{t}}\right)_{t \in[0, T]}$ является $\mathbf{P}^{\lambda}$-мартингалом.

Доказательство см. в [11, гл. VII, §3c, теорема 4].

Обозначим через $D\left(\mathbf{R}_{+}\right)(D([0, T]))$ пространство càdlàg функций (т.е. функций, непрерывных справа и имеющих пределы слева) из $\mathbf{R}_{+}$ (из отрезка $[0, T])$ в $\mathbf{R}$.

Предложение 2.4. Пусть $\mathbf{P}-$ вероятностная мера на пространстве $D\left(\mathbf{R}_{+}\right)$такая, что канонический прочесс $X$ (т.е. прочесс, определяемый по формуле $\left.X_{t}(\omega)=\omega(t)\right)$ является $\mathbf{P}$-прочессом Леви с характеристиками $(b, c, \nu)_{h}$. Пусть $\widetilde{\nu}-$ мера Леви, $\widetilde{\nu} \sim \nu u$

$$
\int_{\mathbf{R}}\left|h(x)\left(1-\frac{d \widetilde{\nu}}{d \nu}(x)\right)\right| \nu(d x)<\infty, \quad \int_{\mathbf{R}}\left(1-\sqrt{\frac{d \widetilde{\nu}}{d \nu}(x)}\right)^{2} \nu(d x)<\infty .
$$

Тогда существует мера $\widetilde{\mathbf{P}} \stackrel{\text { loc }}{\sim} \mathbf{P}($ знак $\stackrel{\text { loc }}{\sim}$ используется для локальной эквивалентности относительно канонической фильтрачии на $\left.D\left(\mathbf{R}_{+}\right)\right)$такая, что $X$ является $\widetilde{\mathbf{P}}$-прочессом Леви с характеристиками $(\widetilde{b}, c, \widetilde{\nu})_{h}$, где $\widetilde{b}=b+\int_{\mathbf{R}} h(x)(\widetilde{\nu}-\nu)(d x)$.

Доказательство см. в [8, теорема 4.39].

3 а м е ч а н и е. Мера Леви $\widetilde{\nu} \sim \nu$, совпадающая с $\nu$ в окрестности нуля, удовлетворяет условиям предложения 2.4.

Локально мартингальной мерой для процесса мы будем называть меру $Q$, эквивалентную исходной, относительно которой процесс является $Q$-локальным мартингалом.

Через $\delta_{a}$ мы будем обозначать дельта-меру в точке $a$.

Лемма 2.1. (i) Пусть $\mathbf{P}$ - вероятностная мера на пространстве $D([0, T])$ или $D\left(\mathbf{R}_{+}\right)$такая, что канонический прочесс $X$ является $\mathbf{P}$-прочессом Леви с характеристиками $\left(b, 0, \alpha \delta_{a}\right)_{0}, b=-\alpha\left(e^{a}-1\right)$. Тогда мера $\mathbf{P}$ - единственная локально мартингальная мера для проиесса $S_{t}=e^{X_{t}}$. Мера $\mathbf{P}$ является также единственной мартингальной мерой длs прочесса $S$. 
(ii) Пусть $\mathbf{P}$ - вероятностная мера на пространстзе $D([0, T])$ или $D\left(\mathbf{R}_{+}\right)$такая, что канонический прочесс $X$ является $\mathbf{P}$-прочессом Леви с характеристиками $(-c / 2, c, 0)_{0}$. Тогда мера $\mathbf{P}-$ единственная локально мартингальная мера для прочесса $S_{t}=e^{X_{t}}$. Mера $\mathbf{P}$ является также единственной мартингальной мерой для прочесса $S$.

Д ок а з а т ель с т в о. Сначала заметим, что, по предложению 2.1 , мера $\mathbf{P}$ в (i) и (ii) является мартингальной для процесса $S$. Далее, если $X \equiv 0$ P-п.н., то утверждения очевидны. Поэтому мы будем предполагать, что $X \not \equiv 0$.

(i) Известно, что $X_{t}=a N_{t}+b t$, где $N-$ пуассоновский процесс с параметром $\alpha$ относительно меры $\mathbf{P}$. По формуле Ито, имеем

$$
d S_{t}=d e^{a N_{t}+b t}=\left(e^{a}-1\right) S_{t-} d N_{t}+b S_{t-} d t=\left(e^{a}-1\right) S_{t-} d\left(N_{t}-\alpha t\right) .
$$

Предположим, что сушествует мера $\mathbf{Q} \sim \mathbf{P}$ такая, что процесс $S$ является Q-локальным мартингалом. Из (2.1) следует, что $\left(N_{t}-\alpha t\right)$ является (с точностью до константы) стохастическим логарифмом от процесса $S$. Значит, $\left(N_{t}-\alpha t\right)$ также является Q-локальным мартингалом (см. [8, гл. II, теорема 8.3]). Поэтому $(\alpha t)$ есть Q-компенсатор точечного процесса $N$. Согласно [8, гл. II, теорема 4.5], процесс $N$ - пуассоновский с параметром $\alpha$ относительно меры $\mathbf{Q}$. Таким образом, распределения канонического процесса по мерам $\mathbf{P}$ и $\mathbf{Q}$ совпадают, т.е. $\mathbf{Q}=\mathbf{P}$.

(ii) Известно, что $X_{t}=\sqrt{c} B_{t}-c t / 2$, где $B-$ броуновское движение относительно меры $\mathbf{P}$. По формуле Ито, имеем

$$
d S_{t}=d e^{X_{t}}=\sqrt{c} S_{t} d B_{t} .
$$

Предположим, что сушествует мера $\mathbf{Q} \sim \mathbf{P}$ такая, что процесс $S$ является Q-локальным мартингалом. Из $(2.2)$ следует, что $B$ также является Q-локальным мартингалом (см. [8, гл. II, теорема 8.3]). Известно, что для любого $t$

$$
\sum_{t_{i-1}, t_{i} \in T^{n}}\left(B_{t_{i}}-B_{t_{i-1}}\right)^{2} \underset{n \rightarrow \infty}{\stackrel{\mathbf{P}}{\longrightarrow}}\langle B\rangle_{t}=t
$$

где $T^{n}=\left\{\left(t_{1}, \ldots, t_{n}\right): 0=t_{1}<\cdots<t_{n}=t\right\}$ и

$$
d\left(T^{n}\right)=\max _{t_{i-1}, t_{i} \in T^{n}}\left|t_{i}-t_{i-1}\right| \underset{n \rightarrow \infty}{\longrightarrow} 0 .
$$

Следовательно,

$$
\sum_{t_{i-1}, t_{i} \in T^{n}}\left(B_{t_{i}}-B_{t_{i-1}}\right)^{2} \underset{n \rightarrow \infty}{\stackrel{\mathrm{Q}}{\longrightarrow}}\langle B\rangle_{t}=t .
$$

Итак, $B$ - непрерывный Q-локальный мартингал с квадратической вариацией $\langle B\rangle_{t}=t$. По теореме Леви, $B$ является Q-броуновским движением. Таким образом, распределения канонического процесса по мерам $\mathbf{P}$ и $\mathbf{Q}$ совпадают, т.е. $\mathbf{Q}=\mathbf{P}$. 
2.2. Сигма-мартингалы. Приводимое ниже определение дано Т. Голлом и Я. Каллсеном в [7].

О п р е д е л е н и е 2.1. Семимартингал $\left(X_{t}\right)_{t \in[0, T]}$ (соответственно $\left.\left(X_{t}\right)_{t \geqslant 0}\right)$ называется $\sigma$-мартингалом, если существует последовательность $\left(D_{n}\right)_{n=1}^{\infty}$ предсказуемых множеств таких, что $D_{n} \subseteq D_{n+1}$, $\bigcup_{n=1}^{\infty} D_{n}=\Omega \times[0, T]$ (соответственно, $\bigcup_{n=1}^{\infty} D_{n}=\Omega \times \mathbf{R}_{+}$) и для любого $n$ стохастический интеграл $\int_{0}^{\bullet} I_{D_{n}} d X_{s}$ является равномерно интегрируемым мартингалом.

3 а м е ч а н и е. Каждый мартингал является $\sigma$-мартингалом.

Предложение 2.5 (Ж.-П. Ансель, К. Стрикер). Пусть $X$ ограниченный снизу $\sigma$-мартингал. Тогда $X$ является локальным мартингалом.

Доказательство см. в [1, следствие 3.5].

2.3. Фундаментальная теорема теории арбитража. Здесь мы приводим традиционную формулировку ФТТА, полученную Ф. Делбаеном и В. Шахермайером. Пусть дана модель

$$
\left(\Omega, \mathscr{F},\left(\mathscr{F}_{t}\right)_{t \geqslant 0}, \mathbf{P},\left(S_{t}\right)_{t \geqslant 0}\right)
$$

Здесь $S-\left(\mathscr{F}_{t}, \mathbf{P}\right)$-семимартингал, отвечающий процессу дисконтированной цены некоторого актива.

О п р е д е лени е 2.2. (i) Cтратегия - это пара $\pi=(x, K)$, где $x \in \mathbf{R}$, а $K=\left(K_{t}\right)_{t \geqslant 0}$ - предсказуемый $S$-интегрируемый процесс (определение интегрируемости см. в [8, гл. III, §6c] или в [13]).

(ii) Дисконтированный капитал стратегии $\pi$ - это процесс $V_{t}=x+$ $\int_{0}^{t} K_{u} d S_{u}$ (определение стохастического интеграла см. в [8, гл. III, §6c] или в [13]).

(iii) Последовательность стратегий $\pi^{n}=\left(x^{n}, K^{n}\right)$ реализует бесплатный ленч с ограниченным риском (free lunch with bounded risk, FLBR), если для их капиталов $V^{n}$ выполняются условия:

(a) $x^{n}=0$ для любого $n$;

(b) существует $a \in \mathbf{R}$ такое, что $\mathbf{P}\left\{\forall t \geqslant 0, V_{t}^{n} \geqslant a\right\}=1$ для любого $n$;

(c) для любого $n$ существует $V_{\infty}^{n}=$ (п.н.) $\lim _{t \rightarrow \infty} V_{t}^{n}$;

(d) существуют $\delta_{1}, \delta_{2}>0$ такие, что $\mathbf{P}\left\{V_{\infty}^{n}>\delta_{1}\right\}>\delta_{2}$ для любого $n$;

(е) для любого $\delta>0 \mathbf{P}\left\{V_{\infty}^{n}<-\delta\right\} \rightarrow 0$ при $n \rightarrow \infty$.

Мы будем также говорить, что $\left\{H^{n}\right\}_{n=1}^{\infty}$ реализует FLBR, если существует $N>0$ такое, что последовательность $\left\{H^{n}\right\}_{n=N}^{\infty}$ реализует FLBR.

Отсутствие бесплатного ленча с ограниченным риском означает, что такой последовательности не существует.

Для модели

$$
\left(\Omega, \mathscr{F},\left(\mathscr{F}_{t}\right)_{t \in[0, T]}, \mathbf{P},\left(S_{t}\right)_{t \in[0, T]}\right)
$$


с конечным временным горизонтом условие отсутствия бесплатного ленча с ограниченным риском определяется аналогично с заменой $V_{\infty}^{n}$ на $V_{T}^{n}$.

Предложение 2.6 (ФТТА). В моделях (2.3) и (2.4) отсутствует бесплатный ленч с ограниченным риском тогда и только тогда, когда $\mathscr{M}_{\sigma} \neq \varnothing$, где $\mathscr{M}_{\sigma}$ определено в (1.3).

Доказательство см. в [6].

\section{3. Модель $S_{t}=e^{X_{t}}$ с конечным временным горизонтом}

В этом разделе мы фиксируем конечный временной интервал $[0, T]$ и рассматриваем модель

$$
\left(\Omega, \mathscr{F},\left(\mathscr{F}_{t}\right)_{t \in[0, T]}, \mathbf{P},\left(S_{t}\right)_{t \in[0, T]}\right),
$$

где $\mathscr{F}_{t}=\mathscr{F}_{t}^{S}, S_{t}=e^{X_{t}}, X=\left(X_{t}\right)_{t \in[0, T]}$ - процесс Леви. Здесь и далее $\left(\mathscr{F}_{t}^{Y}\right)$ означает каноническую фильтрацию процесса $Y$, т.е. $\mathscr{F}_{t}^{Y}=$ $\sigma\left(Y_{u}, u \leqslant t\right)$.

Через $|A|$ мы будем обозначать число элементов в множестве $A$.

3.1. Сигма-мартингальные меры. Следующая теорема утверждает, что «почти всегда» рассматриваемая модель безарбитражна и неполна.

Теорема 3.1. Пусть $X-$ прочесс Леви с характеристиками $(b, c, \nu)_{H}, X$ не является нулевьл прочессом.

(i) Множество $\mathscr{M}_{\sigma}$ пусто тогда и только тогда, когда $X$ является монотонным прочессом.

(ii) Предположим, что $\mathscr{M}_{\sigma}$ непусто. $В$ этом случае $\left|\mathscr{M}_{\sigma}\right|=1$ тогда $u$ только тогда, когда либо $c \neq 0, \nu=0$, либо $c=0, \nu=\alpha \delta_{a}$ (первьй случай отвечает модели Блэка-Шоулса, второй - модели Мертона).

Д ок аза т е л с т в о. (i) Рассмотрим меру Леви

$$
\widetilde{\nu}(d x)=\left(I(|x| \leqslant 1)+e^{-x^{2}} I(|x|>1)\right) \nu(d x) .
$$

По предложению 2.4, существует мера $\widetilde{\mathbf{P}} \sim \mathbf{P}$ такая, что $X$ является $\widetilde{\mathbf{P}}$-процессом Леви с характеристиками $(b, c, \widetilde{\nu})_{H}$. Предположим, что $X$ не монотонен. По предложению $2.2, c \neq 0$ или $c=0$ и есть некоторые ограничения на $b$ и $\widetilde{\nu}$. Легко видеть, что в этих случаях выражение

$$
b+\left(\lambda+\frac{1}{2}\right) c+\int_{\mathbf{R}}\left(e^{\lambda x}\left(e^{x}-1\right)-H(x)\right) \widetilde{\nu}(d x)
$$

положительно для некоторого большого значения $\lambda$ и отрицательно для некоторого маленького значения $\lambda$. Поэтому существует $\lambda$ такое, что выражение в (3.1) равно нулю. Тогда, по предложению 2.3 , мера $\widetilde{\mathbf{P}}^{\lambda_{0}}=$ $k e^{\lambda_{0} X_{T}} \widetilde{\mathbf{P}}$ является мартингальной для процесса $e^{X}$, т.е. $\mathscr{M}_{\sigma} \supseteq \mathscr{M} \neq \varnothing$.

Если $X$ монотонен, то ясно, что $\mathscr{M}_{\sigma}=\varnothing$. 
(ii) Случай 1. Предположим, что $\nu \neq \alpha \delta_{a}$. Очевидно, что существуют две меры $\widetilde{\nu}_{1}$ и $\widetilde{\nu}_{2}$, эквивалентные $\nu$ и совпадающие с $\nu$ в некоторой окрестности нуля, такие, что для любого $\lambda \in \mathbf{R}$

$$
\int_{\mathbf{R} \backslash[-1,1]} e^{\lambda x} \widetilde{\nu}_{i}(d x)<\infty, \quad i=1,2 .
$$

Более того, эти меры могут быть выбраны так, чтобы $\widetilde{\nu}_{1}$ не являлась преобразованием Эшера $\widetilde{\nu}_{2}$, т.е. не существовало бы такого $\lambda$, что $\widetilde{\nu}_{1}(d x)=e^{\lambda x} \widetilde{\nu}_{2}(d x)$.

По предложению 2.4 , существуют меры $\widetilde{\mathbf{P}}_{i}, i=1,2$, эквивалент-

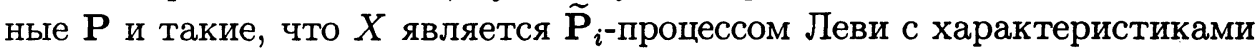
$\left(\tilde{b}_{i}, c, \widetilde{\nu}_{i}\right)_{H}$. Аналогично рассуждениям пункта (i), существуют числа $\lambda_{i}$, $i=1,2$, для которых выполняются условия предложения 2.3, примененного к $\widetilde{\mathbf{P}}_{i}$-процессу Леви $X$. Меры $\widetilde{\mathbf{P}}^{\lambda_{i}}$ являются мартингальными мерами для процесса $e^{X}$. Более того, они не совпадают. Действительно, по [11, гл. VII, § 3c, теоремы 1,2$], X$ есть $\widetilde{\mathbf{P}}^{\lambda_{i}}$-процесс Леви с мерой Леви $\widetilde{\nu}^{\lambda_{i}}(d x)=e^{\lambda_{i} x} \widetilde{\nu}_{i}(d x), i=1,2$. Меры $\widetilde{\nu}^{\lambda_{1}}$ и $\widetilde{\nu}^{\lambda_{2}}$ различны, поскольку $\widetilde{\nu}_{1}$ не является преобразованием Эшера $\widetilde{\nu}_{2}$. Таким образом, $\left|\mathscr{M}_{\sigma}\right| \geqslant|\mathscr{M}|>1$.

Случай 2. Предположим, что $\nu=\alpha \delta_{a}, c \neq 0, \alpha a \neq 0$. Процесс $X$ может быть представлен в виде $X_{t}=b^{\prime} t+\sqrt{c} B_{t}+a N_{t}$, где $b^{\prime}=b-\alpha H(a), B$ - броуновское движение, $N$ - пуассоновский процесс с параметром $\alpha, B$ и $N$ - независимые процессы. Возьмем произвольные $(\lambda, \beta) \in(\mathbf{R},(0, \infty))$. По теореме Гирсанова и предложению 2.4 , существует мера $\mathbf{P}^{\lambda \beta} \sim \mathbf{P}$ такая, что

$$
X_{t}=b^{\prime} t+\sqrt{c}\left(B_{t}^{\lambda \beta}+\lambda t\right)+a N_{t}^{\lambda \beta}
$$

где $B^{\lambda \beta}-\mathbf{P}^{\lambda \beta}$-броуновское движение, $N^{\lambda \beta}-\mathbf{P}^{\lambda \beta}$-пуассоновский процесс с параметром $\beta$. Другими словами, $X$ - это $\mathbf{P}^{\lambda \boldsymbol{\beta}}$-процесс Леви с характеристиками $\left(b^{\prime}+\sqrt{c} \lambda, c, \beta \delta_{a}\right)_{0}$. В силу предложения $2.1, e^{X}$ является $\mathbf{P}^{\lambda \beta}$-мартингалом, если

$$
b^{\prime}+\sqrt{c} \lambda+\frac{c}{2}+\beta\left(e^{a}-1\right)=0 .
$$

Очевидно, что существует много пар чисел $(\lambda, \beta) \in(\mathbf{R},(0, \infty))$ таких, что верно (3.2). Следовательно, $\left|\mathscr{M}_{\sigma}\right| \geqslant|\mathscr{M}|>1$.

Случай 3. Предположим, что либо $\nu=\alpha \delta_{a}, c=0$, либо $\nu=0$, $c \neq 0$. В этом случае единственность $\sigma$-мартингальной меры следует из леммы 2.1 и предложения 2.5 .

Теорема доказана.

3.2. Мартингальные меры. Доказательство теоремы 3.1 показывает, что верно следуюшее утверждение.

Теорема 3.2. Пусть $X$ - прочесс Леви с характеристиками $(b, c, \nu)_{H}, X$ не является нулевьм прочессом. 
(i) Множество $\mathscr{M}$ пусто тогда и только тогда, когда $X$ является монотонным прочессом.

(ii) Предположим, что $\mathscr{M}$ непусто. $B$ этом случае $|\mathscr{M}|=1$ тогда и только тогда, когда либо $c \neq 0, \nu=0$, либо $c=0, \nu=\alpha \delta_{a}$.

3 а м е ч а н и е. Часть (i) теоремы 3.2 была доказана другим методом в $[9$, теорема 1$]$.

\section{4. Модель $S_{t}=e^{X_{t}}$ с бесконечным временным горизонтом}

В этом разделе мы рассматриваем модель

$$
\left(\Omega, \mathscr{F},\left(\mathscr{F}_{t}\right)_{t \geqslant 0}, \mathbf{P},\left(S_{t}\right)_{t \geqslant 0}\right),
$$

где $\mathscr{F}_{t}=\mathscr{F}_{t}^{S}, S_{t}=e^{X_{t}}, X=\left(X_{t}\right)_{t \geqslant 0}$ - процесс Леви.

\section{1. Сигма-мартингальные меры.}

Теорема 4.1. Пусть $X-$ прочесс Леви с характеристиками $(b, c, \nu)_{H}, X$ не является нулевым прочессом.

(i) Множество $\mathscr{M}_{\sigma}$ непусто только в следуюших случаях:

(a) $S$ является $\mathbf{P}$-мартингалом;

(b) $\mathbf{E} S_{1}<1$ и скачки прочесса $X$ не ограничены сверху.

(ii) Предположим, что $\mathscr{M}_{\sigma}$ непусто. В этом случае $\left|\mathscr{M}_{\sigma}\right|=1$ тогда и только тогда, когда либо $c \neq 0, \nu=0$, либо $c=0, \nu=\alpha \delta_{a}$.

Нам будут нужны некоторые вспомогательные утверждения.

Предложение 4.1 (Б. Эссеен). Пусть $\left(\Omega, \mathscr{F},\left(\mathscr{F}_{n}\right)_{n=1}^{\infty}, \mathbf{P}\right)-\oint u \Omega b-$ трованное вероятностное пространство. Предположим, что $\mathscr{F}=$ $\bigvee_{n=1}^{\infty} \mathscr{F}_{n}$. Пусть Q - вероятностная мера на $\mathscr{F}$ такая, ито $\mathbf{Q} \stackrel{\text { loc }}{\sim} \mathbf{P}$, m.е. $\left.\left.\mathbf{P}\right|_{\mathscr{F}_{n}} \sim \mathbf{Q}\right|_{\mathscr{F}_{n}}$ для любого $n \in \mathbb{N}$. Тогда

$$
\frac{\left.d \mathbf{Q}\right|_{\mathscr{F}_{n}}}{\left.d \mathbf{P}\right|_{\mathscr{F}_{n}}} \underset{n \rightarrow \infty}{\stackrel{\mathbf{P} \text {-н.н. }}{\longrightarrow}} \frac{d \mathbf{Q}^{a}}{d \mathbf{P}},
$$

где $\mathbf{Q}^{a}$ - это абсолютно непрерывная часть $\mathbf{Q}$ относительно $\mathbf{P}$.

Доказательство см. в [15, гл. V, теорема 5.2.26].

Лемма 4.1. Пусть $X-$ прочесс Леви с характеристиками $(b, c, \nu)_{H}$,

$$
\tau=\inf \left\{t \geqslant 0: X_{t} \geqslant \ln \frac{3}{2} \text { uлu } X_{t} \leqslant \ln \frac{1}{2}\right\} .
$$

Ecлu $\mathbf{E} e^{X_{1}}>1$, mo $\mathbf{E} e^{X_{\tau}}>1$.

Д о к а за т ел ь с в о. Рассмотрим два случая.

Случай 1. Предположим, что $\mathbf{E} e^{X_{1}}<\infty$. Пусть $N>0$. Известно, что $X=X^{1}+X^{2}$ п.н., где $X^{1}$ и $X^{2}-$ независимые процессы Леви с характеристиками $\left(b^{\prime}, c, \nu^{1}\right)_{H}$ и $\left(0,0, \nu^{2}\right)_{0}$ соответственно, $b^{\prime}=b-\int_{\mathbf{R}} H(x) \nu^{2}(d x), \nu^{1}=\left.\nu\right|_{[-N, N]}, \nu^{2}=\left.\nu\right|_{\mathbf{R} \backslash[-N, N]}$. 
Пусть $\theta-$ момент первого скачка процесса $X^{2}$. Тогда

$$
\begin{aligned}
X_{\tau} & =X_{\tau}^{1} I(\theta>\tau)+\left(X_{\tau}^{1}+X_{\tau}^{2}\right) I(\theta \leqslant \tau), \\
e^{X_{\tau}} & =e^{X_{\tau}^{1}} I(\theta>\tau)+e^{X_{\tau}^{1}+X_{\tau}^{2}} I(\theta \leqslant \tau)
\end{aligned}
$$

и $\mathbf{E} e^{X_{\tau}} \geqslant \mathbf{E} e^{X_{\tau}^{1}} I(\theta>\tau)$. Рассмотрим момент остановки

$$
\tau^{1}=\inf \left\{t \geqslant 0: X_{t}^{1} \geqslant \ln \frac{3}{2} \text { или } X_{t}^{1} \leqslant \ln \frac{1}{2}\right\} \text {. }
$$

Заметим, что $\tau=\tau^{1}$ на множестве $\{\theta>\tau\}$.

Поскольку $X^{1}$ и $X^{2}$ независимы, мы можем предположить, что они заданы на различных вероятностных пространствах $\left(\Omega_{1}, \mathscr{F}_{1}, \mathbf{P}_{1}\right)$ и $\left(\Omega_{2}, \mathscr{F}_{2}, \mathbf{P}_{2}\right)$ соответственно, $\Omega=\Omega_{1} \times \Omega_{2}, \mathbf{P}=\mathbf{P}_{1} \times \mathbf{P}_{2}$. Тогда $\left(\ll \mathbf{E}_{i} »\right.$ означает математическое ожидание по мере $\mathbf{P}_{i}$ )

$$
\begin{aligned}
\mathbf{E} e^{X_{\tau}} & \geqslant \mathbf{E} e^{X_{\tau}^{1}} I(\theta>\tau)=\mathbf{E} e^{X_{\tau^{1}}^{1}} I\left(\theta>\tau^{1}\right) \\
& =\mathbf{E}_{1} \exp \left\{X_{\tau^{1}\left(\omega_{1}\right)}^{1}\left(\omega_{1}\right)\right\} \mathbf{E}_{2} I\left(\theta\left(\omega_{2}\right)>\tau^{1}\left(\omega_{1}\right)\right) \\
& =\mathbf{E}_{1} \exp \left\{X_{\tau^{1}\left(\omega_{1}\right)}^{1}\left(\omega_{1}\right)\right\} \mathbf{P}_{2}\left\{X_{\tau^{1}\left(\omega_{1}\right)}^{2}\left(\omega_{2}\right)=0\right\} \\
& =\mathbf{E} \exp \left\{X_{\tau^{1}}^{1}-\tau^{1} \nu(\mathbf{R} \backslash[-N, N])\right\}
\end{aligned}
$$

Очевидно, что существует $N$ такое, что $\operatorname{E} \exp \left\{X_{1}^{1}-\nu(\mathbf{R} \backslash[-N, N])\right\}>1$. Положим $a=\ln \mathbf{E} \exp \left\{X_{1}^{1}-\nu(\mathbf{R} \backslash[-N, N])\right\}, a>0$. Тогда процесс $M_{t}=\exp \left\{X_{t}^{1}-t \nu(\mathbf{R} \backslash[-N, N])-a t\right\}$ является мартингалом. Процесс $M_{t}^{\tau^{1}}=M_{t \wedge \tau^{1}}$ ограничен и, следовательно, является равномерно интегрируемым мартингалом. Поэтому

$$
\mathbf{E} e^{X_{\tau}} \geqslant \mathbf{E} \exp \left\{X_{\tau^{1}}^{1}-\tau^{1} \nu(\mathbf{R} \backslash[-N, N])\right\}>\mathbf{E} M_{\tau^{1}}=\mathbf{E} M_{0}=1
$$

Случай 2. Предположим, что $\mathbf{E} e^{X_{1}}=\infty$. Известно, что $X=$ $X^{1}+X^{2}$ п.н., где $X^{1}$ и $X^{2}$ - независимые процессы Леви с характеристиками $\left(b, c, \nu^{1}\right)_{H}$ и $\left(0,0, \nu^{2}\right)_{0}$ соответственно, $\nu^{1}=\left.\nu\right|_{[-1,1]}, \nu^{2}=\left.\nu\right|_{\mathbf{R} \backslash[-1,1]}$. Процесс $X^{2}$ является составным пуассоновским процессом, и он может быть представлен в виде $X_{t}^{2}=\sum_{k=1}^{N_{t}} \xi_{k}$, где $N$ - пуассоновский процесс с параметром $\nu^{2}(\mathbf{R}),\left\{\xi_{k}\right\}_{k=1}^{\infty}$ - независимые одинаково распределенные случайные величины с распределением $\nu^{2} / \nu^{2}(\mathbf{R})$. Последовательность $\left\{\xi_{k}\right\}_{k=1}^{\infty}$ не зависит от $N$.

Так как $\mathbf{E} e^{X_{t}^{1}}<\infty$ и $\infty=\mathbf{E} e^{X_{t}}=\mathbf{E} e^{X_{t}^{1}} \cdot \mathbf{E} e^{X_{t}^{2}}$, то $\mathbf{E} e^{X_{t}^{2}}=\infty$. Далее,

$$
\mathbf{E} e^{X_{t}^{2}}=\mathbf{E} \exp \left\{\sum_{k=1}^{N_{t}} \xi_{k}\right\}=\mathbf{E}\left(\mathbf{E} e^{\xi_{1}}\right)^{N_{t}}=\exp \left\{\left(\mathbf{E} e^{\xi_{1}}-1\right) \nu^{2}(\mathbf{R}) t\right\}
$$

откуда $\mathbf{E} e^{\xi_{1}}=\infty$. Пусть $\theta-$ момент первого скачка процесса $X^{2}$ (заметим, что $\left.\Delta X_{\theta}^{2} \geqslant 1>\ln \frac{3}{2}\right), \tau^{1}=\inf \left\{t \geqslant 0: X_{t}^{1} \geqslant \ln \frac{3}{2}\right.$ или $\left.X_{t}^{1} \leqslant \ln \frac{1}{2}\right\}$. 
Имеем

$$
\begin{aligned}
\mathbf{E} e^{X_{\tau}} & =\mathbf{E} e^{X_{\tau^{1}}} I\left(\tau^{1}<\theta\right)+\mathbf{E} e^{X_{\tau}^{1}+X_{\tau}^{2}} I\left(\tau^{1} \geqslant \theta\right) \geqslant \mathbf{E} e^{X_{\tau}^{2}} I\left(\tau^{1} \geqslant \theta\right) \\
& =\mathbf{E} e^{X_{\theta}^{2}} I\left(\tau^{1} \geqslant \theta\right)=\mathbf{E} e^{\xi_{1}} I\left(N_{\tau^{1}} \geqslant 1\right)=\mathbf{E} e^{\xi_{1}} \cdot \mathbf{P}\left\{N_{\tau^{1}} \geqslant 1\right\}=\infty .
\end{aligned}
$$

Лемма доказана.

Следующая лемма будет играть существенную роль в построении мартингальной меры в случае (b) теоремы 4.1 .

Лемма 4.2. Пусть $N \geqslant 1, X$ - прочесс Леви с характеристиками $(b, c, \nu)_{H}$ mакой, что $\mathbf{E} e^{X_{1}}<1 u \nu(a, \infty)>0$ для любого $a>0$. Тогда существует мера Леви $\widetilde{\nu}$ такая, что

i) $\widetilde{\nu} \sim \nu$;

ii) $\left.\tilde{\nu}\right|_{(-\infty, N]}=\left.\nu\right|_{(-\infty, N]}$;

iii) $\tilde{\nu}(\mathbf{R})=\nu(\mathbf{R})$;

iv) $b+c / 2+\int_{\mathbf{R}}\left(e^{x}-1-H(x)\right) \widetilde{\nu}(d x)=0$.

Мера $\widetilde{\nu}$ может быть выбрана не единственньм образом.

Д о к а з а т е л ь с в о. В силу предложения 2.1,

$$
b+\frac{c}{2}+\int_{\mathbf{R}}\left(e^{x}-1-H(x)\right) \nu(d x)<0 .
$$

Возьмем такое $M>N$, что $\nu((N, M))>0$, и выберем такое $a>0$, что

$$
b+\frac{c}{2}+\int_{(-\infty, N]}\left(e^{x}-1-H(x)\right) \nu(d x)+\left(e^{a}-1\right) \nu((N, M))>0 .
$$

Очевидно, что существует такое $\alpha \in(0,1)$, что для меры

$$
\tilde{\nu}=\nu-\left.\alpha \nu\right|_{(N, M)}+\left.\alpha \frac{\nu((N, M))}{\nu((a, \infty))} \nu\right|_{(a, \infty)}
$$

выполняются требуемые в лемме условия.

Ясно, что подобная конструкция позволяет получать различные меры $\tilde{\nu}$, что завершает доказательство.

Лемма 4.3. Пусть $\mathbf{P}$ - вероятностная мера на пространстве $D\left(\mathbf{R}_{+}\right)$такая, что канонический прочесс $X$ - это ненулевой $\mathbf{P}$-прочесс Леви с характеристиками $(b, c, \nu)_{H}$. Пусть $S_{t}=e^{X_{t}}, t \geqslant 0$, u предположим, ито $S$ является $\mathbf{P}$-мартингалом. Тогда мера $\mathbf{P}$ это единственная $\sigma$-мартингальная ( $и$ мартингальная) мера для проиесса $S$ в том и только том случае, когда либо $c \neq 0, \nu=0$, либо $c=0$, $\nu=\alpha \delta_{a}$.

Д ок а за т ел ь с т в о. Если $c \neq 0, \nu=0$ или $c=0, \nu=\alpha \delta_{a}$, то единственность $\sigma$-мартингальной меры следует из леммы 2.1 и предложения 2.5 .

Предположим, что эти условия не выполнены. Пусть

$$
\mathbf{P}_{1}=\operatorname{Law}\left(X_{t} ; t \leqslant 1\right), \quad \mathbf{P}_{2}=\operatorname{Law}\left(X_{t+1}-X_{1} ; t \geqslant 0\right) .
$$


Тогда мера $\mathbf{P}$ - это образ $\mathbf{P}_{1} \times \mathbf{P}_{2}$ при отображении

$$
\begin{aligned}
& D([0,1]) \times D\left(\mathbf{R}_{+}\right) \ni\left(\omega_{1}, \omega_{2}\right) \longmapsto \omega \in D\left(\mathbf{R}_{+}\right), \\
& \omega(t)= \begin{cases}\omega_{1}(t), & t \leqslant 1, \\
\omega_{2}(t-1)+\omega_{1}(1), & t>1 .\end{cases}
\end{aligned}
$$

В силу теоремы 3.1 , существует мера $\widetilde{\mathbf{P}}_{1} \sim \mathbf{P}_{1}$ такая, что $\widetilde{\mathbf{P}}_{1} \neq \mathbf{P}_{1}$

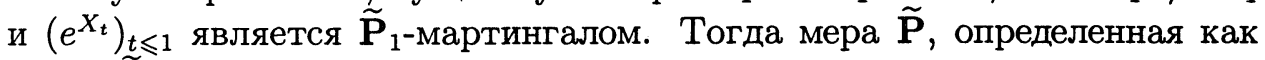
образ $\widetilde{\mathbf{P}}_{1} \times \mathbf{P}_{2}$ при отображении (4.1), является мартингальной мерой для $\left(e^{X_{t}}\right)_{t \geqslant 0}$ и $\tilde{\mathbf{P}} \neq \mathbf{P}$. Лемма доказана.

Док аз а тельст во т е о ремы 4.1. Ясно, что мы можем предположить, что $X$ - это канонический процесс на $D\left(\mathbf{R}_{+}\right)$.

(i) Рассмотрим возможные случаи.

Случай 1. Предположим, что $\mathbf{E} e^{X_{1}}>1$ (в частности, $\mathbf{E} e^{X_{1}}$ может быть бесконечным). Построим последовательность стратегий, которая реализует FLBR. Положим

$$
\begin{aligned}
\tau_{0}=0, \ldots, \tau_{n} & =\inf \left\{t \geqslant \tau_{n-1}:\left|S_{t}-S_{\tau_{n-1}}\right| \geqslant \frac{1}{2} S_{\tau_{n-1}}\right\} \\
& =\inf \left\{t \geqslant \tau_{n-1}: X_{t}-X_{\tau_{n-1}} \geqslant \ln \frac{3}{2} \text { или } X_{t}-X_{\tau_{n-1}} \leqslant \ln \frac{1}{2}\right\} ; \\
K_{t}^{n} & =\frac{1}{n} \sum_{k=1}^{n} \frac{1}{S_{\tau_{k-1}}} I\left(\tau_{k-1}<t \leqslant \tau_{k}\right), \quad n \geqslant 1 .
\end{aligned}
$$

Рассмотрим стратегии $\pi^{n}=\left(0, K^{n}\right)$. Их капиталы имеют вид

$$
V_{t}^{n}=\frac{1}{n} \sum_{k=1}^{n} \frac{S_{\tau_{k} \wedge t}-S_{\tau_{k-1} \wedge t}}{S_{\tau_{k-1}}}, \quad V_{\infty}^{n}=\frac{1}{n} \sum_{k=1}^{n} \frac{S_{\tau_{k}}-S_{\tau_{k-1}}}{S_{\tau_{k-1}}}=\frac{1}{n} \sum_{k=1}^{n} \xi_{k} .
$$

По строго марковскому свойству процессов Леви, $\left\{\xi_{k}\right\}_{k=1}^{\infty}$ являются независимыми одинаково распределенными случайными величинами. По закону больших чисел, $n^{-1} \sum_{k=1}^{n} \xi_{k} \rightarrow \mathbf{E} \xi_{1}$ по вероятности при $n \rightarrow \infty$, а $\mathbf{E} \xi_{1}=\mathbf{E} S_{\tau_{1}}-1$. По лемме 4.1, $\mathbf{E} S_{\tau_{1}}-1>0$. Таким образом, стратегии $\pi^{n}$ реализуют FLBR и, в силу предложения $2.6, \mathscr{M}_{\sigma}=\varnothing$.

Случай 2. Предположим, что $\mathbf{E} e^{X_{1}}=1$. В силу предложения $2.1, e^{X}$ является $\mathbf{P}$-мартингалом, т.е. $\mathbf{P} \in \mathscr{M} \subseteq \mathscr{M}_{\sigma}$.

Случай 3. Предположим, что $\mathbf{E} e^{X_{1}}<1$ и скачки процесса $X$ ограничены сверху. Тогда стратегии $\pi^{n}=\left(0,-K^{n}\right)$, где $K^{n}$ определяются как в случае 1 , реализуют FLBR (заметим, что $\mathbf{E} S_{\tau_{1}}<1$ по теореме об остановке). В силу предложения $2.6, \mathscr{M}_{\sigma}=\varnothing$.

Случай 4. Предположим, что $\mathbf{E} e^{X_{1}}<1$ и скачки процесса $X$ не ограничены сверху. Построим мартингальную меру. Рассмотрим последовательность мер $\left(\mathbf{Q}_{n}\right)_{n=1}^{\infty}$, определяемую на $\left(\mathscr{F}_{n}\right)_{n=1}^{\infty}$ следующей процедурой. 
Шаг 1. В силу леммы 4.2 , существует мера Леви $\nu_{1}$ такая, что $\left.\nu_{1}\right|_{(-\infty, 1]}=\left.\nu\right|_{(-\infty, 1]}, \nu_{1}(\mathbf{R})=\nu(\mathbf{R})$ и существует мера $\widetilde{\mathbf{P}}_{1} \sim \mathbf{P}$, относительно которой $X \sim\left(b, c, \nu_{1}\right)_{H}$ и $e^{X}$ является $\widetilde{\mathbf{P}}_{1 \text {-мартингалом. Обозна- }}$ чим $g_{1}(x)=\ln \frac{d \nu_{1}}{d \nu}(x)$. Очевидно, что $g_{1}(x)=0$ для $x \leqslant 1$. Известно, что процесс плотности меры $\widetilde{\mathbf{P}}_{1}$ относительно меры $\mathbf{P}$ имеет вид

$$
\begin{aligned}
\rho_{t}^{1}(X) & =\exp \left\{\sum_{s \leqslant t} g_{1}\left(\Delta X_{s}\right) I\left(\Delta X_{s} \neq 0\right)+t\left(\nu-\nu_{1}\right)(\mathbf{R})\right\} \\
& =\exp \left\{\sum_{s \leqslant t} g_{1}\left(\Delta X_{s}\right)\right\}, \quad X \in D\left(\mathbf{R}_{+}\right)
\end{aligned}
$$

(см. [10, теорема 33.2]). Положим $\mathbf{Q}_{1}=\left.\rho_{1}^{1} \mathbf{P}\right|_{\mathscr{F}_{1}}$.

Шаа $n$, аде $n \geqslant 2$. Существует мера Леви $\nu_{n}$ такая, что $\nu_{n} \sim \nu$, $\left.\nu_{n}\right|_{(-\infty, n]}=\left.\nu\right|_{(-\infty, n]}, \quad \nu_{n}(\mathbf{R})=\nu(\mathbf{R})$ и для $\nu_{n}$ выполняются условия леммы 4.2. Обозначим $g_{n}(x)=\ln \frac{d \nu_{n}}{d \nu_{n-1}}(x)$. Очевидно, что $g_{n}(x)=0$ для $x \leqslant n-1$. Рассмотрим

$$
\rho_{t}^{n}(X)= \begin{cases}1 & \text { для } t \leqslant n-1, \\ \exp \left\{\sum_{n-1<s \leqslant t} g_{n}\left(\Delta X_{s}\right)\right\} & \text { для } t>n-1\end{cases}
$$

и положим $\mathbf{Q}_{n}=\left.\rho_{n}^{n} \rho_{n}^{n-1} \cdots \rho_{n}^{1} \mathbf{P}\right|_{\mathscr{F}_{n}}$. В силу [10, теорема 33.2], процесс $\left(X_{t}\right)_{t \in[0,1]}$ - это $\mathbf{Q}_{n}$-процесс Леви с характеристиками $\left(b, c, \nu_{1}\right)_{H}, \ldots$, процесс $\left(X_{t+n-1}-X_{n-1}\right)_{t \in[0,1]}$ - это $\mathbf{Q}_{n}$-процесс Леви с характеристиками $\left(b, c, \nu_{n}\right)_{H}$. При этом $\left(e^{X_{t}}\right)_{t \in[0, n]}$ является $\mathbf{Q}_{n}$-мартингалом.

Обозначим $Z_{n}=\rho_{n}^{n} \cdots \rho_{n}^{1}$, тогда $\mathbf{Q}_{n}=\left.Z_{n} \mathbf{P}\right|_{\mathscr{F}_{n}}$. Мы имеем

$$
\begin{gathered}
Z_{n}(X)=\exp \left\{\sum_{s \leqslant 1} \ln \frac{d \nu_{1}}{d \nu}\left(\Delta X_{s}\right)+\sum_{1<s \leqslant 2} \ln \left[\frac{d \nu_{1}}{d \nu}\left(\Delta X_{s}\right) \frac{d \nu_{2}}{d \nu_{1}}\left(\Delta X_{s}\right)\right]+\cdots\right. \\
\left.\quad+\sum_{n-1<s \leqslant n} \ln \left[\frac{d \nu_{1}}{d \nu}\left(\Delta X_{s}\right) \cdots \frac{d \nu_{n}}{d \nu_{n-1}}\left(\Delta X_{s}\right)\right]\right\} \\
=\exp \left\{\sum_{s \leqslant 1} \ln \frac{d \nu_{1}}{d \nu}\left(\Delta X_{s}\right)+\sum_{1<s \leqslant 2} \ln \frac{d \nu_{2}}{d \nu}\left(\Delta X_{s}\right)+\cdots\right. \\
\left.\quad+\sum_{n-1<s \leqslant n} \ln \frac{d \nu_{n}}{d \nu}\left(\Delta X_{s}\right)\right\} \\
=\prod_{s \leqslant 1, \Delta X_{s}>1} \frac{d \nu_{1}}{d \nu}\left(\Delta X_{s}\right) \prod_{1<s \leqslant 2, \Delta X_{s}>2} \frac{d \nu_{2}}{d \nu}\left(\Delta X_{s}\right) \times \cdots \\
\quad \times \prod_{n-1<s \leqslant n, \Delta X_{s}>n} \frac{d \nu_{n}}{d \nu}\left(\Delta X_{s}\right)
\end{gathered}
$$

где $X \in D\left(\mathbf{R}_{+}\right)$. 
Построенные меры $\mathbf{Q}_{n}$ являются согласованными в том смысле, что $\left.\mathbf{Q}_{n}\right|_{\mathscr{F}_{k}}=\mathbf{Q}_{k}$ для любого $k \leqslant n$. Следовательно, сушествует мера $\mathbf{Q}$ такая, что $\left.\mathbf{Q}\right|_{\mathscr{F}_{n}}=\mathbf{Q}_{n}$ для любого $n \in \mathbb{N}$ (см. [4, теорема 1]). Мы утверждаем, что $\mathbf{Q} \sim \mathbf{P}$.

a) Во-первых, докажем, что $\mathbf{P} \ll \mathbf{Q}$. Пусть $X-$ канонический процесс на $D\left(\mathbf{R}_{+}\right)$. Рассмотрим процессы $X_{t}^{2}=\sum_{s \leqslant t} X_{s} I\left(\Delta X_{s}>1\right)$, $X_{t}^{1}=X_{t}-X_{t}^{2}, t \geqslant 0$. Они являются независимыми процессами Леви относительно меры $\mathbf{P}, X^{1} \sim\left(b, c, \nu^{1}\right)_{H}, X^{2} \sim\left(0,0, \nu^{2}\right)_{0}$, где $\nu^{1}=\left.\nu\right|_{(-\infty, 1]}$, $\nu^{2}=\left.\nu\right|_{(1, \infty)}$. Заметим, что $\nu^{2} \neq 0$, поскольку скачки $\Delta X$ не ограничены сверху. Процесс $X^{2}$ является составным пуассоновским процессом и имеет вид $X_{t}^{2}=\sum_{k=1}^{N_{t}} \xi_{k}$, где $N$ - это пуассоновский процесс с параметром $\nu^{2}(\mathbf{R}),\left\{\xi_{k}\right\}_{k=1}^{\infty}$ - независимые одинаково распределенные случайные величины с распределением $\nu^{2} / \nu^{2}(\mathbf{R})$. Последовательность $\left\{\xi_{k}\right\}_{k=1}^{\infty}$ не зависит от $N$.

Для любого $n \in \mathbb{N}$ рассмотрим множество

$$
A_{n}=\left\{\exists s \in(n-1, n]: \Delta X_{s}>n\right\}=\left\{\exists s \in(n-1, n]: \Delta X_{s}^{2}>n\right\} \text { P-п.н. }
$$

Тогда

$$
\mathbf{P}\left(A_{n}\right)=\mathbf{P}\left\{\exists s \leqslant 1: \Delta X_{s}^{2}>n\right\}=\mathbf{E} \mathbf{P}\left(\exists s \leqslant 1: \Delta X_{s}^{2}>n \mid N_{1}\right) .
$$

Мы имеем

$$
\begin{aligned}
& \mathbf{P}\left(\exists s \leqslant 1: \Delta X_{s}^{2}>n \mid N_{1}=k\right)=\mathbf{P}\left(\xi_{1}>n \text { или } \cdots \text { или } \xi_{k}>n \mid N_{1}=k\right) \\
& \quad \leqslant \sum_{i=1}^{k} \mathbf{P}\left\{\xi_{i}>n\right\}=k \mathbf{P}\left\{\xi_{1}>n\right\}=k \frac{\nu^{2}((n, \infty))}{\nu^{2}(\mathbf{R})} .
\end{aligned}
$$

Поэтому

$$
\mathbf{P}\left(A_{n}\right) \leqslant \sum_{k=1}^{\infty} k \frac{\nu^{2}((n, \infty))}{\nu^{2}(\mathbf{R})} \frac{\left(\nu^{2}(\mathbf{R})\right)^{k}}{k !} e^{-\nu^{2}(\mathbf{R})}=\nu^{2}((n, \infty))=\nu((n, \infty)) .
$$

Следовательно,

$$
\sum_{n=1}^{\infty} \mathbf{P}\left(A_{n}\right) \leqslant \sum_{n=1}^{\infty} \nu((n, \infty)) \leqslant \int_{(1, \infty)} x \nu(d x) .
$$

Последнее выражение конечно, поскольку $\int_{(1, \infty)}\left(e^{x}-1\right) \nu(d x)<\infty$ в силу предложения 2.1. По лемме Бореля-Кантелли, $\mathbf{P}\left(\lim \sup _{n \rightarrow \infty} A_{n}\right)=0$. Ввиду (4.2), мы получаем, что для $\mathbf{P}$-п.в. $\omega$ существует $n=n(\omega)$ такое, что для любого $m \geqslant n Z_{m}(\omega)=Z_{n}(\omega)$. Отсюда, в силу предложения 4.1, мы заключаем, что $\mathbf{P} \ll \mathbf{Q}$.

b) Теперь докажем, что $\mathbf{Q} \ll \mathbf{P}$. Рассмотрим те же множества $A_{n}$, что и в случае а). Аналогично, мы получаем

$$
\mathbf{Q}\left(A_{n}\right)=\nu_{n}((n, \infty))=\nu((n, \infty))
$$


(поскольку $\nu_{n}((-\infty, n])=\nu((-\infty, n])$ и $\left.\nu_{n}(\mathbf{R})=\nu(\mathbf{R})\right)$. Следовательно, как и выше, по лемме Бореля-Кантелли, $\mathbf{Q}\left(\lim \sup _{n \rightarrow \infty} A_{n}\right)=0$. Ввиду (4.2) получаем, что для $\mathbf{Q}$-п.в. $\omega$ существует $n=n(\omega)$ такое, что $Z_{m}^{-1}(\omega)=Z_{n}^{-1}(\omega)$ для любого $m \geqslant n$. Отсюда, в силу предложения 4.1 , заключаем, что $\mathbf{Q} \ll \mathbf{P}$.

Мы показали, что мера $\mathbf{Q} \sim \mathbf{P}$. Так как процесс $S$ является Qмартингалом, то $\mathscr{M}_{\sigma} \supseteq \mathscr{M} \neq \varnothing$. Первая часть теоремы доказана.

(ii) Множество $\mathscr{M}_{\sigma}$ непусто только в следующих двух случаях.

Случай 1. Процесс $S$ есть Р-мартингал. Тогда требуемое утверждение - это следствие леммы 4.3.

Cлучай 2. Математическое ожидание $\mathbf{E} S_{1}<1$ и скачки процесса $X$ не ограничены сверху. Тогда из доказательства пункта (i) видно, что можно построить различные мартингальные меры для $e^{X}$.

Теорема 4.1 доказана.

\section{2. Равномерно интегрируемые мартингальные меры.}

Теорема 4.2. Пусть $X$ - ненулевой прочесс Леви. Тогда множество $\mathscr{U} \mathscr{M}$ nусто.

Д оказатель с т о. Поскольку $X \not \equiv 0$, мы имеем, что (п.н.) $\lim \sup _{t \rightarrow \infty}\left|X_{t}\right|=+\infty$. Поэтому, если у процесса $S$ есть Р-п.н. предел $S_{\infty}$, то $S_{\infty}=0$ или $S_{\infty}=\infty \mathrm{P}$-п.н. Следовательно, не существует меры $\widetilde{\mathbf{P}} \sim \mathbf{P}$ такой, что $S$ является $\widetilde{\mathbf{P}}$-равномерно интегрируемым мартингалом.

\section{5. Модель $S_{t}=e^{(X \circ \tau)_{t}}$ с конечным временным горизонтом}

Снова зафиксируем конечный временной интервал $[0, T]$ и рассмотрим модель

$$
\left(\Omega, \mathscr{F},\left(\mathscr{F}_{t}\right)_{t \in[0, T]}, \mathbf{P},\left(S_{t}\right)_{t \in[0, T]}\right),
$$

где $\mathscr{F}_{t}=\mathscr{F}_{t}^{S}, S=e^{(\text {Хот) }}, X=\left(X_{t}\right)_{t \geqslant 0}-$ процесс Леви, $\tau=\left(\tau_{t}\right)_{t \in[0, T]}$ неубывающий càdlàg процесс, не зависящий от $X$.

\section{1. Сигма-мартингальные меры.}

Теорема 5.1. Пусть $X-$ прочесс Леви с характеристиками $(b, c, \nu)_{H}, X$ не является нулевьм прочессом, $\mathbf{P}\left\{\tau_{T}>\tau_{0}\right\}>0$.

(i) Множество $\mathscr{M}_{\sigma}$ пусто тогда и только тогда, когда $X$ является монотонным прочессом.

(ii) Предположим, что $\mathscr{M}_{\sigma}$ непусто. В этом случае $\left|\mathscr{M}_{\sigma}\right|=1$ тогда и только тогда, когда $\tau$ является детерминированным непрерывным прочессом и либо $c \neq 0, \nu=0$, либо $c=0, \nu=\alpha \delta_{a}$.

Д ок аз а т л л с т в о. (i) Поскольку $\tau$ и $X$ - независимые процессы, мы можем предположить, что они заданы на различных вероятностных пространствах $\left(\Omega_{1}, \mathscr{F}_{1}, \mathbf{P}_{1}\right)$ и $\left(\Omega_{2}, \mathscr{F}_{2}, \mathbf{P}_{2}\right)$ соответственно, $\Omega=\Omega_{1} \times \Omega_{2}, \mathbf{P}=\mathbf{P}_{1} \times \mathbf{P}_{2}$. 
Рассмотрим пространство $\left(\Omega_{2}, \mathscr{F}_{2}, \mathbf{P}_{2}\right)$. Можно предположить, что $X$ - канонический процесс на $D\left(\mathbf{R}_{+}\right)$. Если $X$ монотонен, мы получаем, что $\mathscr{M}_{\sigma}=\varnothing$.

Рассмотрим случай, когда $X$ не является монотонным процессом. Доказательство теоремы 3.1 показывает, что для любого $n \in \mathbb{N}$ существует мера $\mathbf{P}_{2}^{n} \sim \mathbf{P}_{2}$ такая, что процесс $\left(e^{X_{t}}\right)_{t \in[0, n]}$ является $\left(\mathscr{F}_{t}^{X}, \mathbf{P}_{2}^{n}\right)$ мартингалом и при этом $\left.\mathbf{P}_{2}^{m}\right|_{\mathscr{F}_{n}^{X}}=\left.\mathbf{P}_{2}^{n}\right|_{\mathscr{F}_{n}^{x}}$ для $m \geqslant n$. Положим

$$
Z_{t}^{n}=\frac{\left.d \mathbf{P}_{2}^{n}\right|_{\mathscr{F}_{t}^{X}}}{\left.d \mathbf{P}_{2}\right|_{\mathscr{F}_{t}^{X}} ^{X}}
$$

Тогда $Z_{t}^{m}=Z_{t}^{n}$ для $0 \leqslant t \leqslant n \leqslant m$. Следовательно, существует процесс $\left(Z_{t}\right)_{t \geqslant 0}$ такой, что $Z_{t}=Z_{t}^{n}$ для $0 \leqslant t \leqslant n$. Процесс $Z$ - строго положительный $\left(\mathscr{F}_{t}^{X}, \mathbf{P}_{2}\right)$-мартингал, $\mathbf{E}_{\mathbf{P}} Z_{t}=1$, произведение $Z e^{X}-$ также $\left(\mathscr{F}_{t}^{X}, \mathbf{P}_{2}\right)$-мартингал (см. [8, гл. III, предложение 3.8$\left.]\right)$.

Из независимости $X$ и $\tau$ мы получаем, что $\mathbf{E}_{\mathbf{P}} Z_{\tau_{T}}=1$ и $\mathbf{P}\left\{Z_{\tau_{T}}>0\right\}=1$. Следовательно, $\widetilde{\mathbf{P}}:=Z_{\tau_{T}} \mathbf{P}-$ это вероятностная мера, эквивалентная $\mathbf{P}$.

Для любого $s \leqslant t$

$$
\mathbf{E}_{\mathbf{P}_{2}} Z_{t} e^{X_{s}}=\mathbf{E}_{\mathbf{P}_{2}} \mathbf{E}_{\mathbf{P}_{2}}\left(Z_{t} e^{X} \mid \mathscr{F}_{s}^{X}\right)=\mathbf{E}_{\mathbf{P}_{2}} Z_{s} e^{X_{s}}=1,
$$

следовательно, для любого $u \in[0, T]$ мы имеем

$$
\mathbf{E}_{\widetilde{\mathbf{P}}} S_{u}=\mathbf{E}_{\mathbf{P}_{1}} \mathbf{E}_{\mathbf{P}_{2}} Z_{\tau_{T}\left(\omega_{1}\right)} e^{X_{\tau_{u}\left(\omega_{1}\right)}}=1 .
$$

Для любых $s_{1} \leqslant \cdots \leqslant s_{n} \leqslant s \leqslant t \leqslant w$ и любых $A_{1}, \ldots, A_{n} \in \mathscr{B}(\mathbf{R})$

$$
\begin{aligned}
& \mathbf{E}_{\mathbf{P}_{2}} Z_{w} e^{X_{t}} I\left(e^{X_{s_{1}}} \in A_{1}, \ldots, e^{X_{s_{n}}} \in A_{n}\right) \\
& \quad=\mathbf{E}_{\mathbf{P}_{2}} Z_{t} e^{X_{t}} I(\cdots)=\mathbf{E}_{\mathbf{P}_{2}} Z_{s} e^{X_{\bullet}} I(\cdots)=\mathbf{E}_{\mathbf{P}_{2}} Z_{w} e^{X_{s}} I(\cdots),
\end{aligned}
$$

следовательно, для любых $u_{1} \leqslant \cdots \leqslant u_{n} \leqslant u \leqslant v$ и любых $A_{1}, \ldots, A_{n} \in$ $\mathscr{B}\left(\mathbf{R}_{+}\right)$мы имеем

$$
\begin{aligned}
& \mathbf{E}_{\widetilde{\mathbf{P}}} S_{v} I\left(S_{u_{1}} \in A_{1}, \ldots, S_{u_{n}} \in A_{n}\right) \\
& \quad=\mathbf{E}_{\mathbf{P}_{1}} \mathbf{E}_{\mathbf{P}_{2}} Z_{\tau_{T}\left(\omega_{1}\right)} e^{X_{\tau_{v}\left(\omega_{1}\right)}} I\left(e^{X_{\tau_{u_{1}}\left(\omega_{1}\right)}} \in A_{1}, \ldots, e^{\left.X_{\tau_{u_{n}}\left(\omega_{1}\right)} \in A_{n}\right)}\right. \\
& \quad=\mathbf{E}_{\mathbf{P}_{1}} \mathbf{E}_{\mathbf{P}_{2}} Z_{\tau_{T}\left(\omega_{1}\right)} e^{X_{\tau_{u}\left(\omega_{1}\right)}} I(\cdots)=\mathbf{E}_{\widetilde{\mathbf{P}}} S_{u} I\left(S_{u_{1}} \in A_{1}, \ldots, S_{u_{n}} \in A_{n}\right) .
\end{aligned}
$$

Вместе с леммой о монотонных классах (или теоремой о $\pi$ - $\lambda$-системах, см. $\left[12\right.$, гл. II, $\S 2$, теорема 2]) это дает, что $S$ является $\left(\mathscr{F}_{t}^{S}, \widetilde{\mathbf{P}}\right)$-мартингалом.

(ii) Из приведенной выше конструкции меры $\widetilde{\mathbf{P}}$ видно, что если существует много мартингальных мер для процесса $e^{X}$, то существует много мартингальных мер для процесса $e^{(X \circ \tau)}$. Следовательно, в силу теорем 3.1 и 3.2 , если $\tau$ - детерминированный и непрерывный процесс, 
то $\left|\mathscr{M}_{\sigma}\right|=|\mathscr{M}|=1$ тогда и только тогда, когда либо $c \neq 0, \nu=0$, либо $c=0, \nu=\alpha \delta_{a}$.

Если $\tau$ - детерминированный и разрывный процесс, то существует $t_{0}$ такое, что $\tau_{t_{0}-}=u<v=\tau_{t_{0}}$. Пусть $\widetilde{\mathbf{P}}=Z_{\tau_{T}} \mathbf{P}-$ это мера, определенная в (i). Тогда $\widetilde{\mathbf{P}}$ является мартингальной мерой для процесса $S$. Ясно, что процесс $X$ имеет $\widetilde{\mathbf{P}}$-независимые приращения. Существует $\sigma\left(X_{v}-X_{u}\right)$-измеримая случайная величина $\rho \neq 1$ такая, что $\rho>0$ P-п.н. и

$$
\mathbf{E}_{\mathbf{P}} e^{X_{v}-X_{u}} \rho Z_{\tau_{t_{0}}}=1, \quad \mathbf{E}_{\mathbf{P}} \rho Z_{\tau_{t_{0}}}=1
$$

(например, мы можем найти $\rho$ в виде $\rho=a_{1} I_{A_{1}}+a_{2} I_{A_{2}}+a_{3} I_{A_{3}}+$ $I_{\mathbf{R} \backslash\left(A_{1} \cup A_{2} \cup A_{3}\right)}$, где $a_{i}>0, A_{i}$ - непересекающиеся множества и $\left.\mathbf{P}_{2}\left(A_{i}\right)>0, i=1,2,3\right)$.

Рассмотрим меру $\widetilde{\mathbf{P}}^{\prime}=\rho Z_{\tau_{T}} \mathbf{P}$. Очевидно, что $e^{(\text {Х })}$ является $\widetilde{\mathbf{P}}^{\prime}$-мартингалом. Поэтому $\left|\mathscr{M}_{\sigma}\right| \geqslant|\mathscr{M}|>1$.

Предположим, что $\tau$ - недетерминированный процесс. Тогда мы можем найти меру $\mathbf{P}_{1}^{\prime} \sim \mathbf{P}_{1}, \mathbf{P}_{1}^{\prime} \neq \mathbf{P}_{1}$. Пусть $Z$ - это процесс, определенный в (i). Меры $\widetilde{\mathbf{P}}=Z_{\tau_{T}} \mathbf{P}_{1} \times \mathbf{P}_{2}$ и $\widetilde{\mathbf{P}}^{\prime}=Z_{\tau_{T}} \mathbf{P}_{1}^{\prime} \times \mathbf{P}_{2}$ являются мартингальными для процесса $S$, т.е. $\left|\mathscr{M}_{\sigma}\right| \geqslant|\mathscr{M}|>1$.

Теорема доказана.

5.2. Мартингальные меры. Доказательство теоремы 5.1 показывает, что верно следующее утверждение.

Теорема 5.2. Пусть $X$ - прочесс Леви с характеристиками $(b, c, \nu)_{H}, X$ не является нулевым прочессом, $\mathbf{P}\left\{\tau_{T}>\tau_{0}\right\}>0$.

(i) Множество $\mathscr{M}$ пусто тогда и только тогда, когда $X$ является монотонньм прочессом.

(ii) Предположим, что $\mathscr{M}$ непусто. $B$ этом случае $|\mathscr{M}|=1$ тогда и только тогда, когда $\tau$ является детерминированным непрерывным, прочессом и либо $c \neq 0, \nu=0$, либо $c=0, \nu=\alpha \delta_{a}$.

\section{6. Модель $S_{t}=e^{(X \circ \tau)_{t}}$ с бесконечным временным горизонтом}

В этом разделе мы рассматриваем модель

$$
\left(\Omega, \mathscr{F},\left(\mathscr{F}_{t}\right)_{t \geqslant 0}, \mathbf{P},\left(S_{t}\right)_{t \geqslant 0}\right),
$$

где $\mathscr{F}_{t}=\mathscr{F}_{t}^{S}, S=e^{(X \circ \tau)_{t}}, X=\left(X_{t}\right)_{t \geqslant 0}-$ процесс Леви, $\tau=\left(\tau_{t}\right)_{t \geqslant 0}$ неубывающий càdlàg процесc, не зависящий от $X$.

Мы исследуем только существование и единственность равномерно интегрируемой мартингальной меры.

Теорема 6.1. Пусть $\tau_{\infty}=\infty \mathbf{P}-$ п.н., $X$ - ненулевой прочесс Леви. Тогда множество $\mathscr{U} \mathscr{M}$ nусто.

Этот результат получается аналогично теореме 4.2. 
Благодарность. Я благодарен А.С. Черному за руководство этой работой и за многочисленные замечания и дополнения, которые сушественно улучшили текст статьи. Я благодарен А.Н. Ширяеву за важные комментарии и предложения, позволившие, в частности, сократить доказательство теоремы 3.1 .

\section{СПИСОК ЛИТЕРАТУРЫ}

1. Ansel J.-P., Stricker C. Couverture des actifs contingents et prix maximum. - Ann. Inst. H. Poincaré, 1994, v. 30, № 2, p. 303-315.

2. Barndorff-Nielsen O. E., Shephard N. Modelling by Lévy processes for financial econometrics. - Lévy Processes. Theory and Applications. Ed. by O. E. Barndorff-Nielsen et al. Boston: Birkhäuser, 2001, p. 283-318.

3. Carr P., Geman H., Madan D., Yor M. Stochastic volatility for Lévy processes. Math. Finance, 2003, v. 13, № 3, p. 345-382.

4. Черный $A$. C. Семейства согласованных вероятностных мер. - Теория вероятн. и ее примен., 2001, т. 46 , в. 1 , с. 118-121.

5. Delbaen $F$., Schachermayer $W$. A general version of the fundamental theorem of asset pricing. - Math. Ann., 1994, v. 300, № 3, p. 463-520.

6. Delbaen $F$., Schachermayer $W$. The fundamental theorem of asset pricing for unbounded stochastic processes. - Math. Ann., 1998, v. 312, № 2, p. 215-250.

7. Goll T., Kallsen J. A complete explicit solution to the log-optimal portfolio problem. - Ann. Appl. Probab., 2003, v. 13, № 2, p. 774-799.

8. Jacod J., Shiryaev A. N. Limit Theorems for Stochastic Processes. Berlin: SpringerVerlag, 2003, $661 \mathrm{p}$.

9. Jakubenas $P$. On option pricing in certain incomplete markets. - Труды МИАН, 2002 , т. 237 , c. $123-142$.

10. Sato K.-I. Lévy Processes and Infinitely Divisible Distributions. Cambridge: Cambridge Univ. Press, 1999, $486 \mathrm{p}$.

11. Ширяев $A$. . Н. Основы стохастической финансовой математики. Т. 2. М.: Фазис, 1998, 544 c.

12. Ширяев А. Н. Вероятность. М.: Наука, 2004, 927 с.

13. Ширяев $A . H$., Черный A.C. Векторный стохастический интеграл и фундаментальные теоремы теории арбитража. - Труды МИАН, 2002, т. 237, с. 12-56.

14. Sin C. A. Strictly local martingales and hedge ratios on stochastic volatility models. Ph. D. Dissertation. Ithaka, NY: Cornell University, Graduate School, 1996.

15. Stroock $D$. W. Probability Theory. An Analytic View. Cambridge: Cambridge Univ. Press, 2000, $536 \mathrm{p}$. 